\title{
ON THE DEVELOPMENT OF NEW VUV AND UV SOLID STATE LASER SOURCES FOR PHOTOCHEMICAL APPLICATIONS
}

\author{
A. C. CEFALAS, M. A. DUBINSKII*, E. SARANTOPOULOU, \\ R. Yu. ABDULSABIROV*, S. L. KORABLEVA*, A. K. NAUMOV*, \\ V. V. SEMASHKO* and C. A. NICOLAIDES \\ National Hellenic Research Foundation Theoretical and Physical Chemistry \\ Institute 48 Vas. Constantinou Aven. Athens 116-35, Greece \\ *Kazan State University, 18 Lenin Str. 420008 Kazan, Russia.
}

(Received 17 July, 1992)

\begin{abstract}
Laser action in the VUV and UV region of the spectrum at $172 \mathrm{~nm}$ and at $260 \mathrm{~nm}$ had been obtained from $\mathrm{LaF}_{3}: \mathrm{Nd}^{3+}$ and $\mathrm{LiYF}_{4}: \mathrm{Nd}^{3+}$ crystals respectively when they were pumped with an $\mathrm{F}_{2}$ pulsed discharge molecular laser at $157 \mathrm{~nm}$.

The fluorescence spectrum of $\mathrm{LiYF}_{4}: \mathrm{Ho}^{3+}$ monocrystals using the same pumping source has been obtained as well and it suggests that this crystal can be used for generating tunable coherent VUV and UV radiation. This finding opens new prospects for the development of a new class of solid state VUV and UV laser sources for photochemical applications.
\end{abstract}

KEY WORDS: Laser Chemistry, Tunable solid-state lasers, Interconfigurational ef transitions.

\section{INTRODUCTION}

Vacuum ultraviolet (VUV) and ultraviolet (UV) laser sources are useful devices for a variety of applications ranging from photochemistry ${ }^{1}$ to photolithography. ${ }^{2}$ Especially in the VUV region of the spectrum only very few laser devices operate with limited tunability. Besides the existing laser sources, tunable and coherent VUV radiation has been obtained with a variety of methods such as frequency mixing in gases and metal vapours. ${ }^{3,4}$ In addition to the above methods tunable VUV radiation can in principle be obtained from a wide class of solid-state dielectric crystals activated by rare-earth ions ${ }^{5}$ due to the allowed $d-f$ radiative transitions.

Laser action from $\mathrm{LaF}_{3}: \mathrm{Nd}^{3+}(\mathrm{LaF}: \mathrm{Nd})$ at $172 \mathrm{~nm}$ has been achieved previously by Waynant and Klein. ${ }^{6,7}$ The crystal was pumped by the VUV fluorescence of excited $\mathrm{Kr}_{2}{ }^{*}$ dimers (excited by an e-beam). However the method of excitation of the LaF:Nd crystal was too complicated to be used on a small laboratory scale. Recently $^{8}$ we have managed to achieve laser action from the same crystal using as 
a pumping source an $F_{2}$ pulsed discharge molecular laser operating at $157 \mathrm{~nm}$. In this paper we report on the efficient laser action from LaF:Nd and $\mathrm{LiYF}_{4}: \mathrm{Nd}^{3+}$ (YLF:Nd) single crystals around $172 \mathrm{~nm}$ and $260 \mathrm{~nm}$ respectively.

It is evident that the realization of this new pumping scheme opens the way for the wide use of rare-earth activating ions in dielectric crystals for efficient generation of coherent VUV and UV radiation. Therefore the search for new rare earth activated wide band-gap dielectric crystals to be used as potential active media for solid state lasers is of great importance and in this paper we report as well on the interconfigurational $4 \mathrm{f}^{9} 5 \mathrm{~d}-4 \mathrm{f}^{10}$ VUV and UV fluorescence. of YLF: $\mathrm{Ho}^{3+}$ crystals under irradiation at $157 \mathrm{~nm}$ from an $\mathrm{F}_{2}$ pulsed discharge molecular laser.

The broad band features of the fluorescence in the VUV and UV regions of the spectrum and the photochemical stability of these crystals under $F_{2}$ laser pumping suggest that these materials can be used as active media for VUV and UV tunable laser sources.

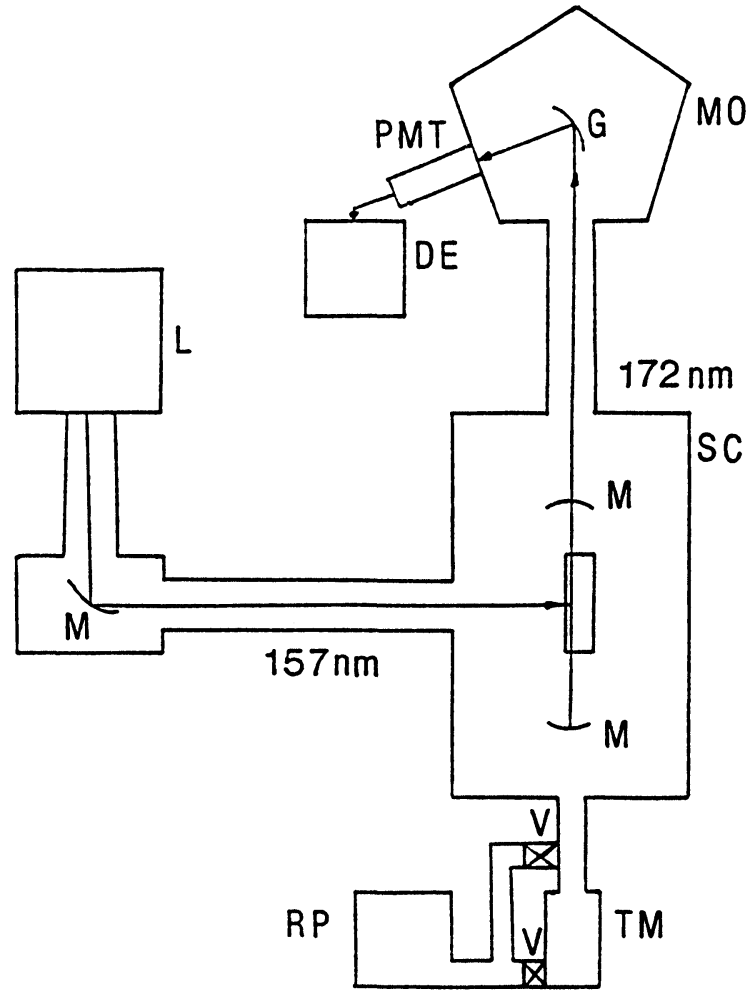

Figure 1 Experimental apparatus for obtaining laser action from $\mathrm{LaF}_{3}: \mathrm{Nd}^{3+}$ and $\mathrm{LiYF}_{4}: \mathrm{Nd}^{3+}$ crystals at 172 and $260 \mathrm{~nm}$ respectively. L: $F_{2}$ pulsed discharge molecular laser at $157 \mathrm{~nm}, \mathrm{M}$ : mirrors, SC: stainless steel vacuum chamber, MO: VUV monochromator, PMT: solar blind photomultiplier, DE: detection electronics, G: grating 1200 lines/mm. TM: turbo molecular pump, RP: rotary pump, V: valve. 


\section{EXPERIMENTAL}

The experimental apparatus is indicated in Figure 1. The laser crystals were cylindrical rods $50 \mathrm{~mm}$ long with $3 \mathrm{~mm}$ radius and they were placed inside an optical cavity consisted of two mirrors been $150 \mathrm{~mm}$ apart. The back mirror was totally reflecting at $172 \mathrm{~nm}$ and $260 \mathrm{~nm}$ and the front mirrors were $65 \%$ transparent at $172 \mathrm{~nm}$ and at $260 \mathrm{~nm}$. The laser beam from the $F_{2}$ laser was focused on the crystals using an off axis concave mirror at right angles. A polished flat window was made on the side of the cylindrical surface of the crystal for efficient excitation of the pumped medium. The maximum energy density of the pumping source on the surface of the crystal was $120 \mathrm{~mJ} / \mathrm{cm}^{2}$. The optical path of the laser radiation from the pumping source at $157 \mathrm{~nm}$ and the excited solid-state laser was kept within stainless steel vacuum lines at $10^{-5} \mathrm{mbar}$ using a turbo molecular pump. The laser output from the solid-state laser was detected using a VUV monochromator with resolution of 0.1 $\mathrm{nm}$ and a solar blind photomultiplier. Signals were recorded using a Tektronix 7104 fast oscilloscope and a box-car integrator interfaced to a computer. The $F_{2}$ pump laser has been described previously9,10 and it can deliver $12 \mathrm{~mJ}$ (as it is measured with a Gentek PR100-type pyroelectric detector) in a single pulse with $12 \mathrm{~ns}$ at FWHM.

\section{RESULTS AND DISCUSSION}

\section{a) $\mathrm{LaF}_{3}: \mathrm{Nd}^{3+}$}

Laser action from the LaF:Nd crystal at $172 \mathrm{~nm}$ has been obtained when the crystal was pumped at $157 \mathrm{~nm}$. The concentration of the $\mathrm{Nd}^{3+}$ ions was 0.5 at $\%$. The pumping threshold for obtaining stimulated emission was $3 \mathrm{~mJ}$.

Stimulated emission from the LaF:Nd crystal was observed previously by Waynant $^{7}$ at 0.1 at $\%$ concentration of the $\mathrm{Nd}$ ion. The differences between our and Waynant's data are attributed to the different pumping geometry. The transverse pumping geometry of our experiment requires the active medium to have higher absorption coefficients of the pumping radiation, which are unattainable at low doping levels. The temporal evolution of the pumping pulse at $157 \mathrm{~nm}$ and the excited laser pulse at $172 \mathrm{~nm}$ is indicated in Figure 2. Saturation in the Laf:Nd-laser output vs input dependence is taking place, Figure 3 , while the mechanisms of this process are probably similar to those that occur for the $\mathrm{YLF}: \mathrm{Ce}^{3+}$ laser, ${ }^{11}$ such as formation of transient colour centers by excited state absorption of the pumping radiation. The laser output at $172 \mathrm{~nm}$ was due to the transitions from the $4 \mathrm{f}^{2} 5 \mathrm{~d}$ mixed configuration, Figure 4 , to the ${ }^{4} \mathbf{I}_{11 / 2,13 / 2}$ levels of the $4 f^{3}$ configuration of the $\mathbf{N d}^{3+}$ ion.

\section{3b) $\mathrm{LiYF}_{4}: \mathrm{Nd}^{3+}$}

Laser action at $260 \mathrm{~nm}$ has been obtained from the YLF:Nd crystal as well using the same excitation geometry and optical cavity as for the LaF:Nd crystal. The 


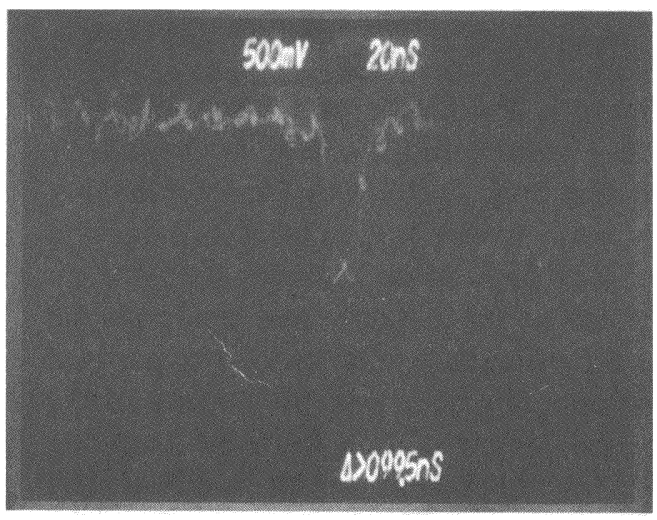

Figure 2a Temporal evolution of the pumping pulse at $157 \mathrm{~nm}$.

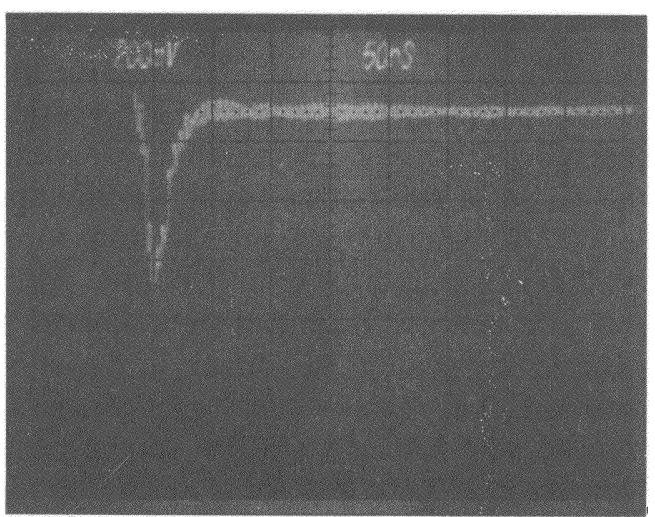

Figure 2b Temporal evolution of the excited laser pulse at $172 \mathrm{~nm}$ from $\mathrm{LaF}_{3}: \mathrm{Nd}^{3+}$.

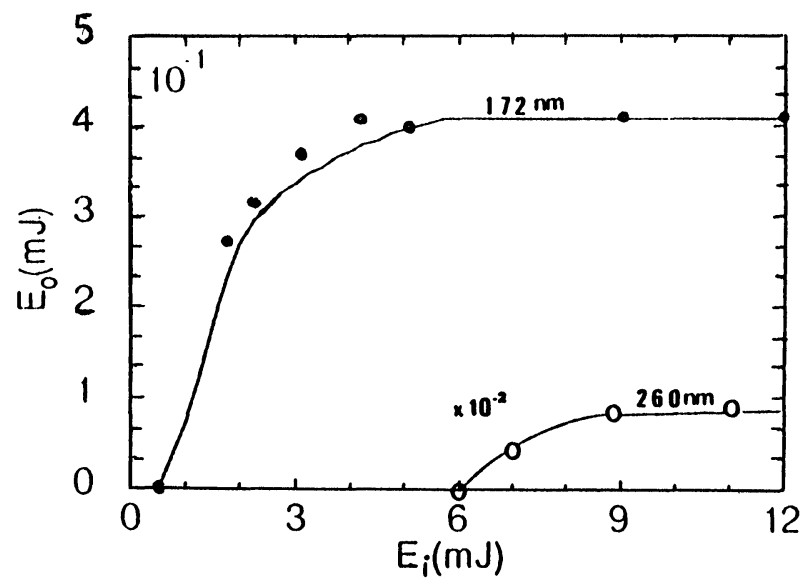

Figure 3 Output energy $E_{o}$ at $172 \mathrm{~nm}$ - and at $260 \mathrm{~nm}$ o as a function of the input $E_{i}$ of the pumping laser pulse at $157 \mathrm{~nm}$.

fluorescence spectrum for this crystal is indicated in Figure 5. Three peaks have been identified in the VUV region of the spectrum around 182, 186 and $196 \mathrm{~nm}$. Emission at these wavelengths corresponds to transitions between the lowest levels of the excited $4 \mathrm{f}^{2} 5 \mathrm{~d}$ mixed configuration and the lowest ground state levels of $4 \mathrm{f}^{3}$ configuration, Figure 6 . The peak around $182 \mathrm{~nm}$ corresponds to the transition between the levels of $4 f^{2} 5 d$ mixed configuration and the ${ }^{4} I_{9 / 2}$ ground state level of the $4 \mathrm{f}^{3}$ configuration. Similarly the two peaks at 186 and at $196 \mathrm{~nm}$ correspond to transitions between the $4 \mathrm{f}^{2} 5 \mathrm{~d}$ mixed configuration and the ${ }^{4} \mathrm{I}_{11 / 2}$ and ${ }^{4} \mathrm{I}_{13 / 2}$ levels of the $4 \mathrm{f}^{3}$ configuration. The peak around $260 \mathrm{~nm}$ can be assigned to transitions between the levels of $4 f^{2} 5 d$ mixed configuration and the ${ }^{2} G_{7 / 2,5 / 2}$ and ${ }^{2} H_{11 / 2}$ levels of $4 f^{3}$ single configuration. The threshold for laser action for this crystal was $6 \mathrm{~mJ}$ of pumping energy and the maximum output at $260 \mathrm{~nm}$ for 0.5 at $\%$ concentration of the $\mathrm{Nd}^{3+}$ ion was $1 \mu \mathrm{J}$. 


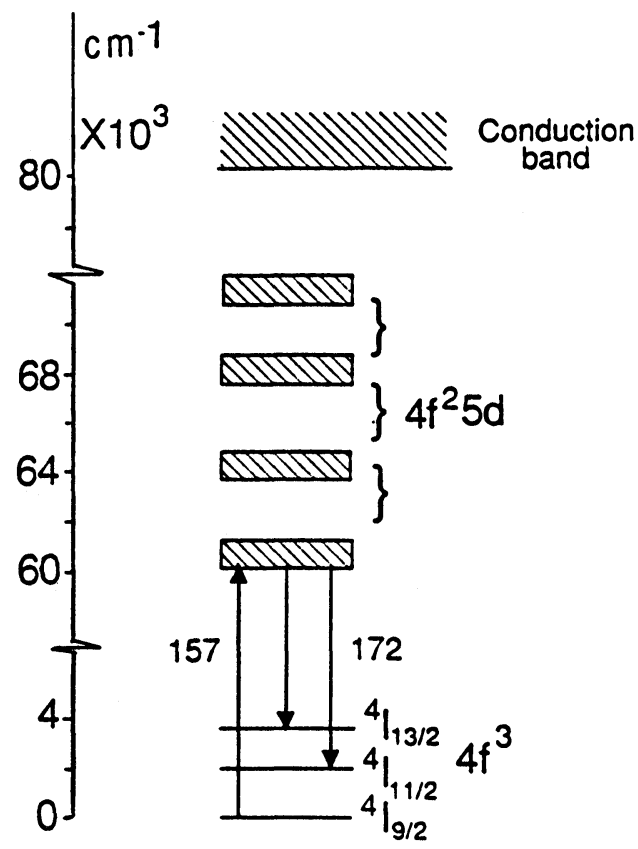

Figure 4 Simplified energy diagram of the $\mathrm{Nd}^{3+}$ ions in the $\mathrm{LaF}_{3}$ host.

$\frac{150 \quad 181}{212}$

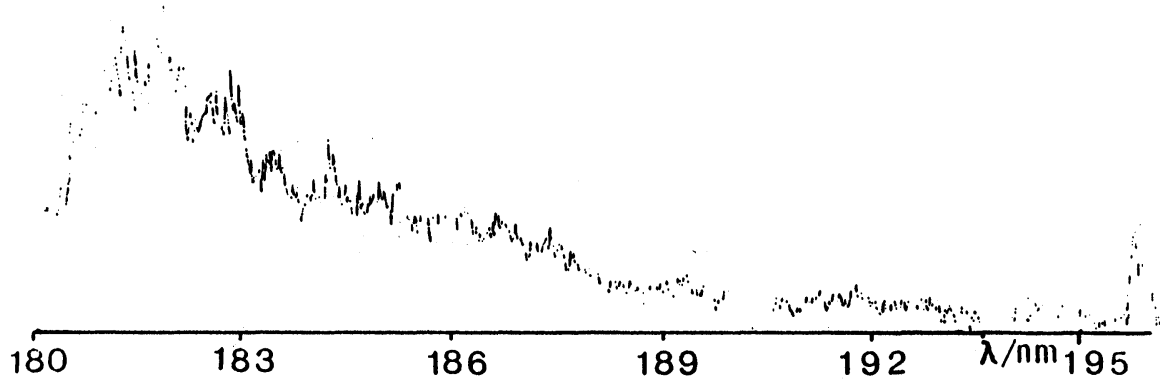

Figure 5 Fluorescence spectrum of the $\mathrm{LiYF}_{4}: \mathrm{Nd}^{3+}$ under $\mathrm{F}_{2}$ laser pumping 

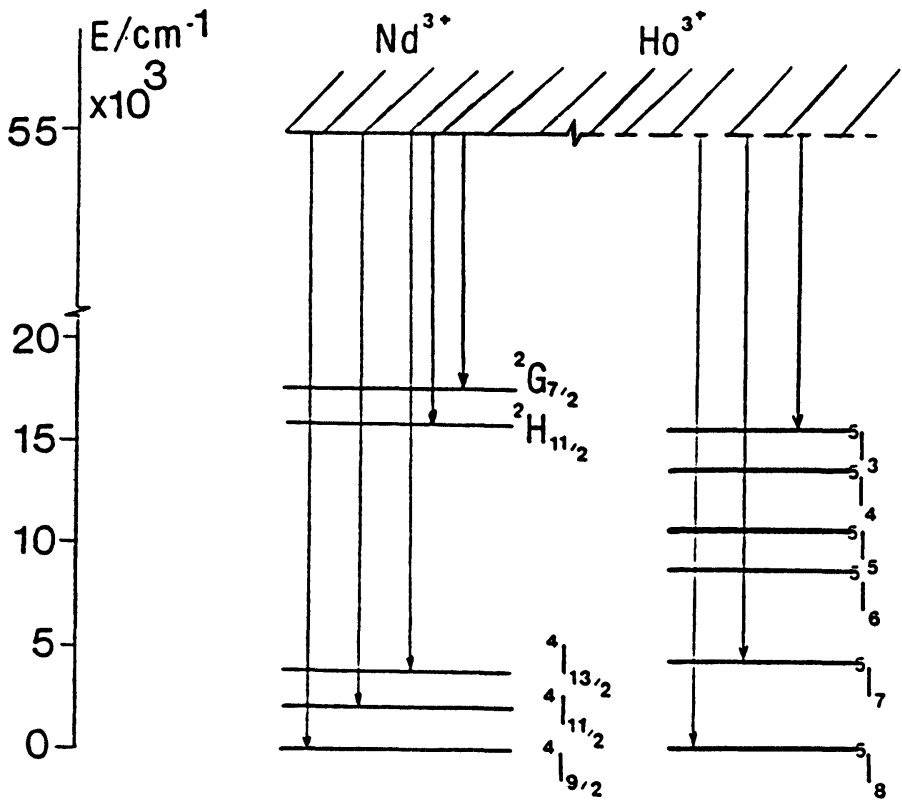

Figure 6 Simplified energy diagram of $\mathrm{Nd}^{3+}$ ions in $\mathrm{LiYF}_{4}$ host.

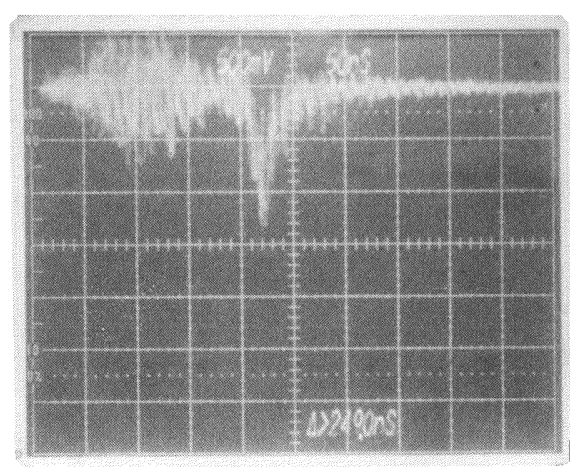

Figure 7 Temporal evolution of the excited laser pulse at $260 \mathrm{~nm}$ under $\mathrm{F}_{2}$ laser pumping of $\mathrm{LiYF}_{4}: \mathrm{Nd}^{3+}$ crystal.

The temporal evolution of the excited laser pulse is indicated in Figure 7. 


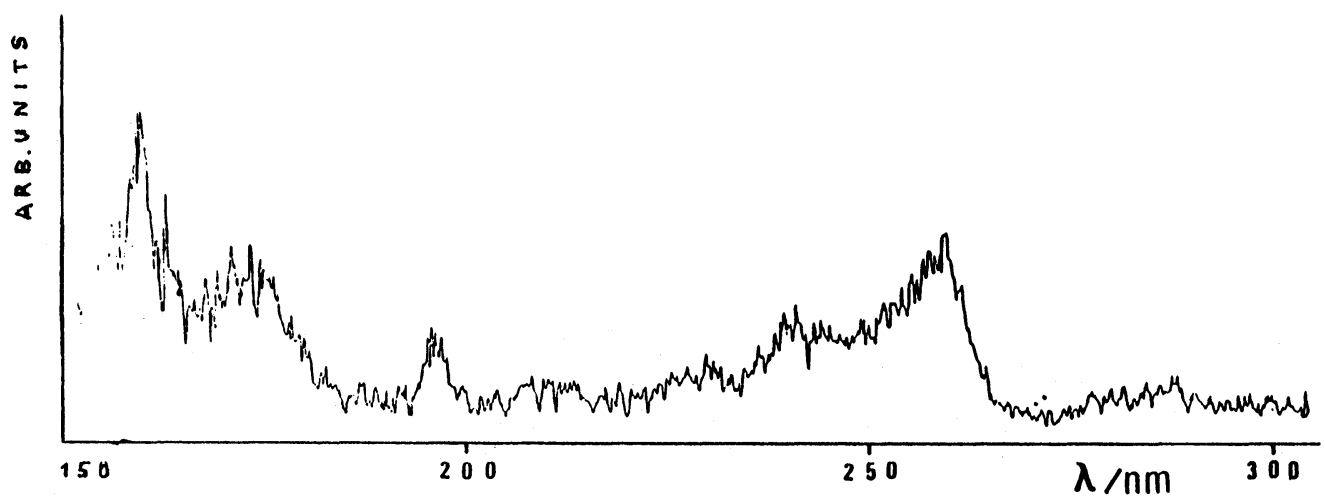

Figure 8 Fluorescence spectrum of $\mathrm{LiYF}_{4}: \mathrm{Ho}^{3+}$ under $\mathrm{F}_{2}$ laser pumping.

\section{3c) $\mathrm{LiYF}_{4}: \mathrm{Ho}^{3+}$}

The laser induced fluorescence spectrum of some rare earth activated YLF crystals have been studied in the spectral region between $157-300 \mathrm{~nm}$. The short pulse duration of the exciting laser pulse with respect to the measured fluorescence lifetime for all the samples on the one hand and the fact that the YLF:Ho ${ }^{3+}$ crystals absorb strongly at $157 \mathrm{~nm}$ on the other hand, suggest that the molecular $F_{2}$ laser source can efficiently pump these crystals for generating VUV and UV radiation.

The fluorescence spectrum of $\mathrm{YLF}: \mathrm{Ho}^{3+}$ crystal under the $\mathrm{F}_{2}$ laser pumping is indicated in Figure 8. Four broad fluorescence peaks have been observed around 170, 194, 240 and $258 \mathrm{~nm}$. Emission at 170 and $194 \mathrm{~nm}$ can be tentatively assigned to transitions between the lowest levels of the excited $4 \mathrm{f}^{9} 5 \mathrm{~d}$ mixed configuration and the ${ }^{5} \mathrm{I}_{6}$ and ${ }^{5} \mathrm{~F}_{5}$ levels of $4 \mathrm{f}^{3}$ configuration. The broad bands around 240 and $258 \mathrm{~nm}$ can be assigned to transitions between the $4 \mathrm{f}^{9} 5 \mathrm{~d}$ mixed configuration and the ${ }^{5} \mathrm{G}_{4}$ and ${ }^{5} \mathrm{G}_{3,2}$ levels of $4 \mathrm{f}^{3}$ single configuration of $\mathrm{Ho}^{3+}$ ion.

The fluorescence spectrum remains unchanged over 10 hours of constant laser irradiation at $157 \mathrm{~nm}$ with $10 \mathrm{~Hz}$ repetition rate.

The absence of any visible colouration was encouraging for the crystal growth of this material to be used as VUV and UV active laser medium.

\section{CONCLUSIONS}

Laser action at $172 \mathrm{~nm}$ from $\mathrm{LaF}_{3}: \mathrm{Nd}^{3+}$ and at $260 \mathrm{~nm}$ from YLF:Nd crystals have been obtained when these crystals were pumped with an $\mathrm{F}_{2}$ pulsed discharge laser at $157 \mathrm{~nm}$. The rare-earth activated dielectric crystals such as YLF:Ho offer the possibility to be used as an active media in tunable VUV and UV laser sources for photochemical applications. 


\section{References}

1. C. Skordoulis, E. Sarantopoulou, S. M. Spyrou and A. C. Cefalas. Z. Phys., D. Atoms Molecules and Clusters 18, 175 (1991).

2. Y. Takakuwa, M. Niwano, S. Fujita, Y. Takeda and N. Miyamoto. Appl. Phys. Lett., 58, 1635 (1991).

3. T. Efthimiopoulos, B. P. Stoichef and R. I. Thomson. Opt. Lett., 14, 624 (1989).

4. J. C. Miller, R. N. Compton and C. P. Cooper. J. Chem. Phys., 76, 3967 (1982).

5. K. H. Yang and J. A. De Luca. Appl. Phys. Lett., 29, 499 (1976).

6. R. W. Waynant and P. H. Klein. Appl. Phys. Lett., 46, 14 (1985).

7. R. W. Waynant. Appl. Phys., B. 828, 205 (1982)

8. M. A. Dubinskii, A. C. Cefalas and C. A. Nicolaides. Opt. Comm., 88, 122 (1992).

9. C. Skordoulis, E. Sarantopoulou, S. M. Spyrou and A. C. Cefalas. J. Mod. Opt., 37, 501 (1990).

10. C. Skordoulis, S. M. Spyrou and A. C. Cefalas. Appl. Phys., B. 51, 141 (1990).

11. D. F. Ehrlich, P. J. Moulton and R. M. Osgood. Opt. Lett., 4, 184 (1979). 